\title{
DESARROLLO DE LA INVESTIGACIÓN: UNA MIRADA AL BASAMENTO LEGAL ANGOLANO
}

\author{
Josefina Castillero Velásquez* \\ https://orcid.org/0000-0001-9303-4952 \\ Elexis Craib Díaz** \\ http://orcid.org/0000-0002-1437-0833 \\ Eurico Wongo Gungula*** \\ http://orcid.org/0000-0002-5685-1328
}

RECIBIDO: Octubre 2021 / ACEPTADO: Diciembre 2021 / PUBLICADO: Enero 2022

Como citar: Castillero Velásquez, Josefina; Craib Díaz, Elexis; Gungula, Eurico. (2022). Desarrollo de la investigación: una mirada al basamento legal angolano. Telos: revista de Estudios Interdisciplinarios en Ciencias Sociales, 24 (1), Venezuela. (Pp. 195-207).

DOI: www.doi.org/10.36390/telos 241.12

\section{RESUMEN}

El desarrollo de la investigación desde contextos universitarios angolanos ha sido intencionado paulatinamente, para lo cual existen diversos pronunciamientos ministeriales. No obstante las investigaciones generadas desde contextos universitarios angolanos, precisan perfeccionar su dinámica. Esa problemática estimuló una indagación a partir del siguiente objetivo: analizar el basamento legal angolano en relación al desarrollo de la investigación y su implementación en contextos universitarios entre 2005 y 2020. Este estudio fue desarrollado mediante métodos analítico-hermenéuticos, aplicados en revisiones documentales de documentos legales tales como: estrategias, programas, reglamentos, decretos, estatutos, artículos y libros; a partir de criterios relacionados con los aspectos estratégicos, operativos y de estructura organizacionalfuncional, lo cual permitió estudiar políticas educativas, normativas institucionales y procesos universitarios. Los principales referentes teóricos fueron: Fuentes (2004), Tobón (2005), Gungula; Castillero; Mendes (2017); Gungula; Suárez; Artigas (2020) y Gungula; Artigas; Faustino (2020); quienes abordaron diversos enfoques epistemológicos relacionados con el desarrollo de la investigación. Los resultados alcanzados indican la existencia de intenciones ministeriales hacia el desarrollo de la investigación, posibilitando la creación de infraestructuras y procesos investigativos; no obstante persisten las dificultades al planificar, organizar, ejecutar, controlar y evaluar las investigaciones, donde se hace necesaria la sistematización de las

\footnotetext{
*Universidad Óscar Ribas, Angola, Máster en Psicología. E-mail: kaluajoo@hotmail.com

** Universidad Óscar Ribas, Angola, PhD en Ciencias Pedagógicas. E-mail: craibelexis@gmail.com

**** Universidad Óscar Ribas, Angola, PhD en Ciencias Pedagógicas. E-mail: euricowongowongo@gmail.com
} 
competencias investigativas en los profesores, que le permitan desarrollar dinámicamente las investigaciones.

Palabras clave: proyecciones ministeriales, Actividades de Investigación, Gestión, Competencias Investigativas, Contexto universitario angolano.

\title{
Development of the investigation: a look at the angolan legal base
}

\begin{abstract}
The development of research from Angolan university contexts has been gradually intentional, for which there are various ministerial pronouncements. However, the investigations generated from Angolan university contexts need to improve their dynamics. This problem stimulated an investigation based on the following objective: analyze the Angolan legal basis in relation to the development of research and it is implementation in university contexts between 2005 and 2020 . This study was developed using analytical-hermeneutical methods, applied in documentary reviews of legal documents such as: strategies, programs, regulations, decrees, statutes, articles and books; based on criteria related to strategic, operational and organizational-functional structure aspects, which allowed studying educational policies, institutional regulations and university processes. The main theoretical references were: Fuentes (2004), Tobón (2005), Gungula; Castillero; Mendes (2017); Gungula; Suárez; Artigas (2020) and Gungula; Artigas; Faustino (2020); who approached various epistemological approaches related to the development of research. The results obtained indicate the existence of ministerial intentions towards the development of the investigation, enabling the creation of research infrastructures and processes; however, the difficulties persist when planning, organizing, executing, controlling and evaluating investigations, where it is necessary to systematize the investigative competencies in teachers, which allow them to dynamically develop investigations.
\end{abstract}

Keywords: Ministerial projections, Research activities, Management, Investigative Competences, Angolan university context.

\section{Introducción}

El desarrollo de la investigación en contextos universitarios angolanos ha sido un proceso intencionado hacia su dinamización desde el Ministerio de Educación Superior, Ciencia, Tecnología e Innovación. Esta intencionalidad parte desde la existencia de basamentos legales hasta su implementación en procesos formativos y de investigación (Artigas; Gungula, 2020).

El Ministerio de Educación Superior, Ciencia, Tecnología e Innovación en Angola, estableció entre sus objetivos, realizar procesos formativos interrelacionados con la investigación científica, que contribuyeron al desarrollo sustentable del país mediante la ejecución de diagnósticos, estudios teórico-descriptivos, valoraciones epistemológicas, proyecciones teórico-prácticas, implementaciones proactivas, intervenciones comunitarias, corroboraciones científicas, generalizaciones, así como también socializaciones.

La realización de procesos formativos interrelacionados con la investigación científica, necesitaron de la sistematización de los resultados investigativos por las Instituciones de Educación Superior; desde la reflexión sobre los procesos de creación e innovación y la 
estructuración de diversos subprocesos, como alternativas para la construcción de conocimientos científicos relevantes con potencialidades de generalización hacia otros contextos universitarios, a escala global (Gungula; Suárez; Artigas, 2020).

Sin embargo, en el contexto universitario angolano existieron insuficiencias teóricometodológicas al planificar, organizar, ejecutar, controlar e evaluar los procesos investigativos, donde los profesores presentaron dificultades al desarrollar las competencias investigativas desde la concepción estratégica de investigaciones, la organización científica de proyectos de investigación, el desarrollo proactivo del sistema de actividades científicas, el control y regulación de la actividad investigativa, así como también la valoración de los impactos de las investigaciones científicas.

Las insuficiencias teórico-metodológicas presentes en los procesos investigativos en el contexto universitario angolano, requieren de un análisis de sus diferentes tendencias de desarrollo, fragilidades, estrategias y el impacto del acelerado desarrollo tecnológico actual en su transformación, rumbo a la calidad (Gungula; Castillero; Mendes, 2017; Gungula; Artigas; Faustino, 2020).

Las dificultades que existieron en los procesos investigativos en el contexto angolano, fueron motivadas además por limitaciones cognitivo-procedimentales, al elaborar e implementar investigaciones. Lo cual reveló un problema, delimitado hacia insuficiencias presentes, al gestionar competencias investigativas desde contextos universitarios angolanos.

El actual estudio de las dificultades que existieron en los procesos investigativos en el contexto angolano, transitó por el análisis de estrategias, programas, reglamentos, decretos, estatutos, artículos y libros; donde se interpretaron políticas educativas, lineamientos pedagógicos, normas reguladoras, parámetros, reformas, modelos para las investigaciones científicas, así como también los procesos o sistemas formativos, lo cual fue explicitado en las siguientes secciones:

- Basamento legal angolano para el desarrollo de la investigación

- Desarrollo de la investigación en contextos universitarios angolanos

- Gestión de competencias investigativas en contextos universitarios angolanos

Las secciones anteriormente mencionadas, se desarrollaron mediante una indagación teórico-descriptiva, teniendo como objetivo: analizar el basamento legal angolano en relación al desarrollo de la investigación y su implementación en contextos universitarios entre 2005 y 2020; mediante el método analítico-hermenéutico, aplicado a través de revisiones documentales de documentos legales tales como: estrategias, programas, reglamentos, decretos, estatutos, artículos y libros; a partir de criterios relacionados con los aspectos estratégicos, operativos y de estructura organizacional-funcional.

El análisis del basamento legal angolano en relación al desarrollo de la investigación y su implementación en contextos universitarios entre 2005 y 2020, permitió mostrar cronológicamente proyecciones ministeriales, estructuración e dinamización institucional de procesos y actividades dirigidas hacia la investigación, revelando al unísono las irregularidades organizativo-procedimentales, que se manifestaron en contextos universitarios.

\section{Basamento legal angolano para el desarrollo de la investigación}

Finalizadas las acciones del conflicto armado angolano, el sistema educativo entre 1995 - 2005, transitó por una reestructuración, adecuando sus procesos al nuevo contexto 
nacional, frente a carencias tales como infra-estructura, equipamientos, medios técnicos, bibliografía y personal docente.

Mientras, la Educación Superior se desarrollaba desde una universidad estatal ("Agostinho Neto") y otras universidades privadas, donde se comenzaron a identificar las necesidades de formar un profesional con competencias científico-técnicas, que desarrollara investigaciones, proporcionando alternativas de solución a los problemas sociales.

Al identificar la necesidad de formar profesionales universitarios con competencias investigativas, fueron estimulados conjuntamente los proyectos y cooperaciones nacionales e internacionales, donde se facilitaron informaciones actualizadas, que elevaban paulatinamente los niveles científico-tecnológicos (Ministério da Educação e Cultura, 2001).

No obstante, el subsistema Enseñanza Superior fue perfeccionado a partir del 2005, al delimitarse diversas líneas maestras, para mejorar la gestión universitaria, asumiendo un carácter profesionalizante, donde era necesario contar con especialistas capacitados en planificación, estadística, economía, diseño curricular, evaluación académica, metodologías cualitativo-cuantitativas y uso de las tecnologías (Secretaria de Estado do Ensino Superior, 2005).

Además fueron adoptadas medidas para mejorar los recursos materiales como bibliografía, laboratorios e implementos tecnológicos en apoyo a las actividades académicoinvestigativas. Lo cual proporcionó el desarrollo de prácticas profesionales, asistencia técnica, colaboraciones, foros regulares, seminarios, conferencias, exposiciones y jornadas comunitarias abiertas.

Posteriormente en 2009, fue emitido un decreto por el Consejo de Ministros, que estableció normas generales reguladoras del subsistema de Enseñanza Superior, donde se explicitaba, la libertad académica como una autonomía capaz de asegurar diferentes métodos, permitiendo desarrollar procesos cognitivos, investigativos y extensionistas; lo cual estimuló la creación de condiciones que aseguraran la producción continua del conocimiento, las innovaciones científicas y tecnológicas, el apoyo al desarrollo comunitario, la difusión de informaciones institucionales sobre los respectivos cursos, así como también la creación de mecanismos de evaluación externa sobre la calidad de los servicios universitarios (Conselho de Ministros, 2009).

Así se fueron incentivando diversos procesos investigativos en contextos universitarios. Los cuales, a partir del 2011 también fueron reglamentados ministerialmente (Ministerio Educación Superior, Ciencia e Tecnología), donde aparecieron varios requisitos, como la aprobación de Consejos Científicos, autonomías investigativas, así como también proyectos educativos y culturales (Assembleia Nacional, 2001).

La aprobación de los Consejos Científicos en contextos universitarios también transitó por un proceso reglamentario, donde fueron reconocidos como órganos colegiales consultivos. Los cuales posteriormente llegaron a definir las políticas científicas a desarrollar en los espacios formativos, investigativos y extensionistas, mediante planes para ejecutar pesquisas, deliberación sobre la creación, transformación o extinción de áreas departamentales o unidades de investigación y la propuesta de tribunales para los cursos de post-grado (Angola, 2011a).

Además los Consejos Científicos posibilitaron la aprobación de programas, proyectos, reglamentos, contratos y protocolos con instituciones nacionales o extranjeras; que permitieron crear o reorganización las instituciones vinculadas a pesquisas, donde se definieron líneas 
científicas a desarrollar en laboratorios, centros o institutos. Por otra parte incidieron en proceso direccionados hacia el apoyo financiero para desarrollar eventos, así como también la atribución de premios al mérito científico (Angola, 2015a).

Al unísono otras políticas, reconocidas gubernamentalmente, consideraron las acciones investigativas, como determinantes del progreso socio-económico; lo que estimuló la aprobación de una estrategia nacional para incentivar procesos científico-tecnológicos, incidiendo en áreas tales como: educación, cultura, formación profesional, enseñanza superior, agricultura-pesca, telecomunicaciones-tecnologías de Información, industria-petróleo-gasrecursos minerales, salud, recursos hidráulicos, energía y ambiente.

En esta Estrategia Nacional de Ciencia, Tecnología e Innovación se reconoció, que la misión primaria para las instituciones universitarias e investigativas, es preparar al ciudadano para responder con eficacia y eficiencia a los desafíos contemporáneos; donde es posible perfeccionar conocimientos, facilitar soluciones tecnológicas, aumentar producciones, satisfacer necesidades sociales, así como también sustentar el desarrollo socio-económico.

Esta Estrategia Nacional de Ciencia, Tecnología e Innovación para Angola delimitó entre sus objetivos generales: capacitar recursos humanos, crear bases materiales, proporcionar la cooperación científico-tecnológica, institucionalizar, promover culturas científicas, responder a cuestiones de defensa, seguridad, sociedad, cultura y medio ambiente; además estimuló el desarrollo de estructuras económico-empresariales, dinamizando la ciencia, tecnología e innovación (Angola, 2011c).

Luego fue estructurándose una coordinación estratégica del Sistema Nacional de Ciencia, Tecnología e Innovación, donde la actividad investigativa y el desarrollo tecnológico en Angola eran gestionados por un departamento ministerial, órganos consultivos e instituciones especializadas. Mientras, fueron establecidos mecanismos en diferentes instituciones investigativas (público-privadas); donde se explicitaron las pertinencias científico-técnicas, contextualizaciones y cooperaciones desde ejes estratégicos tales como: recursos humanosformación, desarrollo de programas-proyectos sectoriales, movilización-programación presupuestaria, organización de la cooperación, innovación-transferencia tecnológica, asesoría y evaluación (Angola, 2011 b).

Esas acciones de coordinación proporcionaron además a nivel nacional, condiciones esenciales para institucionalizar el Consejo Superior de Ciencia, Tecnología e Innovación, como órgano multisectorial consultivo, donde se orientaban disímiles políticas estratégicas; que permitieron analizar estudios, emitir valoraciones, proponer soluciones y estimular la gestión científica.

Posteriormente fue aprobado el reglamento del Consejo Superior de Ciencia, Tecnología e Innovación, donde se tuvo la intención de ejecutar procesos científicos mediante, estrategias, proyectos, programas 0 acciones investigativas; sustentados en acompañamientos metodológicos, que garantizaban elevar la calidad y los impactos económico-socioculturales (Angola, 2014).

El Consejo Superior de Ciencia, Tecnología e Innovación en Angola, tuvo además como atribuciones: proponer políticas, estrategias y mecanismos para coordinar nacionalmente el desarrollo científico-tecnológico, presentar planes, evaluar ejecuciones político-estratégicas, pronunciarse sobre los financiamientos públicos destinados a instituciones relacionadas con desarrollos de la ciencia, tecnología e innovación, posibilitar relaciones entre investigaciones 
científicas, viabilizar el desarrollo tecnológico, la innovación y los sectores productivos, así como también promover excelencia en procesos científico-tecnológicos desde la educación (Angola, 2014).

Consecutivamente fue definido el plan anual de ciencia, tecnología e innovación, direccionado a resolver problemas socio-económicos; mediante proyectos orientados a crear programas para la formación postgraduada (Maestría, Doctorados, Post-Doctorados), el desarrollo de las tecnologías ambientales e investigaciones (médicas, ética, bioética, bioseguridad, responsabilidad social, biodiversidad); potenciando la utilización sustentable de recursos naturales, el estudios de suelos, uso de las TIC, la divulgación científica, la capacitación de recursos humanos, la estructuración de fondos bibliográficos y la transferencia tecnológica (Angola, 2015b).

Asimismo, surgieron varias instituciones públicas de investigación científica, desarrollo tecnológico e innovación, que se direccionaban hacia la enseñanza, indagación, divulgación, financiamiento, fiscalización y evaluación, sustentado por principios tales como: autonomía, buenas prácticas, optimización de recursos disponibles, formación de recursos humanos, promoción de culturas científico-tecnológicas, planificación por objetivos y cooperación interinstitucional (Angola, 2015c).

Entre tanto, fue redimensionada la Educación Superior a partir del 2016, al ser reconocida como un conjunto de órganos, instituciones, disposiciones y recursos, que posibilitaron formar cuadros directivos, realizar investigaciones e intervenciones comunitarias, para contribuir con el desarrollo nacional, asegurando una preparación científica, técnica, cultural y humana (Angola, 2016).

Entre los principales objetivos que tuvo la Educación Superior Angolana a partir del 2016, estuvieron las promociones de investigaciones científicas, innovaciones y aplicaciones de los resultados científicos. Lo cual estimuló nuevos roles docente, desde intencionalidades socioindagativas, al implementarse actividades educativas, programas, proyectos científicos, planes e intervenciones sociales, que proporcionaron desarrollos multidimensionales al país (Angola, 2016).

Conjuntamente fueron identificadas en 2016, diversas responsabilidades científicotecnológicas, direccionadas al fortalecimiento de la ciencia, técnica y tecnología; ofreciendo diversas soluciones a los problemas económico-socioculturales. Lo que visionó adecuados desempeños profesionales, promoviendo investigaciones, desarrollos, transferencia tecnológica e innovación.

Los nuevos roles y responsabilidades docentes relacionados con la actividad investigativa a partir del 2018, fueron reconocidos como deberes caracterizados por el estudio, indagación, aplicación de tecnologías, innovación y producción científica; los cuales se socializan en debates, seminarios, talleres, conferencias, congresos, coloquios, así como también desde revistas científicas nacionales e internacionales (Angola, 2018).

Posteriormente, fue creada la Academia Angolana de Ciencias (AAC); teniendo como función social, promover pesquisas fundamentadas en procesos científicos. Para lograr esa misión fueron trazados los siguientes objetivos: reconocer el conocimiento científico, estimular la concientización pública, premiar la excelencia científica desde servicios de consultoría teóricometodológica, divulgar las buenas prácticas científicas a partir de la ética profesional y propiedad intelectual; así como también promover la colaboración nacional e internacional (Angola, 2020). 
En el período transcurrido entre 2005 y 2020 el subsistema de Enseñanza Superior en Angola contó con un basamento legal, que sustentó las políticas relacionadas con el desarrollo de la investigación, lo cual reveló las intenciones ministeriales hacia el desarrollo de la investigación, posibilitando la creación de infraestructuras y procesos investigativos en contextos universitarios.

\section{Desarrollo de la investigación en contextos universitarios angolanos}

El contexto universitario angolano entre los años 2005-2020, transitó por perfeccionamientos sistemáticos en estructuras y procesos donde se estimuló el desarrollo de la investigación. No obstante se manifestaron disímiles insuficiencias al gestionar adecuadamente la investigación científica, desde subprocesos de institucionalización, financiamientos, gestión de currículos, estructuración de claustros docentes, gestión de matrículas, armonización curricular, formaciones post-graduadas, desarrollo de estructuras físico-tecnológicas, así como también productividad científica.

Los procesos de institucionalización en los centros universitarios, presentaron omisiones de visión, concepciones, planificación, delimitación de actores (promotores, gestores, ejecutores, técnicos, beneficiarios), roles o competencias profesionales y articulación armoniosa; lo que limitó la calidad en los procesos formativos, académico, investigativo y extensionista (Secretaria de Estado do Ensino Superior, 2005).

Mientras, los financiamientos presentaron limitaciones motivadas por su naturaleza, carácter, movimientos, disponibilidad, fuentes 0 fondos desde las estructuras universitarias (públicas, privadas, público-privadas), donde se desarrollaban diferentes gestiones financieras, cobro de tasas sobre diversos servicios prestados, así como también donaciones nacionales e internacionales, incidiendo en los procesos académicos, investigativos y extensionistas (Secretaria de Estado do Ensino Superior, 2005).

Conjuntamente al gestionar currículos, se produjeron alteraciones en las unidades curriculares, provocando notables distorsiones en sus proyecciones iniciales, influyendo en la dosificación del contenido, distribución de la carga docente, desarrollo de la dimensión investigativa, ejecución de la práctica pre-profesional, la contextualización de los conocimientos, así como también la implementación de concepciones epistemológicas y praxiológicas (Secretaria de Estado do Ensino Superior, 2005).

Además al estructurar los claustros docentes universitarios, se presentaron irregularidades en cuanto a los perfiles académicos, nivel científico, competencias indagativas, experiencias pedagógicas, proyección educativa, así como también presencialidad; lo que incidió directamente en el desarrollo de objetivos formativos, actividades académicoinvestigativas, extensión universitaria e intervención comunitaria.

Asimismo, al gestionar matrículas se presentaron irregularidades en relación a la formación vocacional, adecuación del perfil de formación básica a las exigencias universitarias (candidatos a instituciones privadas, público-privadas), motivación, adaptación y contextualización; lo cual incidió en el desarrollo de las actividades académico-investigativas.

Simultáneamente, el desarrollo de la investigación en el contexto universitario angolano manifestó otras irregularidades influenciadas por la armonización curricular, las formaciones post-graduadas, los procesos dirigidos hacia la evaluación, acreditación y certificación universitaria, así como también por las condiciones y estructuras físico-tecnológicas 
(servicios informáticos); lo que incidió directamente en el desarrollo de objetivos formativos, actividades académico-investigativas y la productividad científica.

La armonización curricular fue un aspecto esencial, debido a las diferentes estructuras curriculares del subsistema Educación Superior, que incidieron en la paridad entre cursos del mismo perfil profesional, contextualización, realización de actividades prácticas, respuestas al desarrollo socio-económico, movilidad docente-estudiantil y el desarrollo de la investigación (Gungula; Castillero; Mendes, 2017).

Las formaciones de pre-grado y post-grado también presentaron limitaciones provocadas por la gestión en los programas de formación, eficiencia formativa, (tiempo de formación y cierre en los ciclos de estudios), preparación de proyectos para crear cursos, sistema de información y financiamiento; lo cual limitó el desarrollo de la investigación e innovación (MESCTI, 2019). Estas limitaciones de desarrollo de cursos de post-grado no solo se ven en Africa, paises latinoamericanos las siguen enfrentando, obligando a hacer convenios entre paises para incrementar la formación en estos niveles, cuestión que pudiese ser tomada por Angola como ejemplo (Ortiz Carrillo, 2021).

Además existieron dificultades en el funcionamiento de las estructuras físicotecnológicas relacionadas con servicios informáticos (programas, software, herramientas digitales, instalaciones, conectividad, redes), siendo imprescindibles para la gestión académicocientífica desarrollada por gestores, docentes, investigadores, funcionarios no docentes y estudiantes; lo cual incidió directamente en los niveles de productividad científica.

La productividad científica además estuvo limitada debido a los financiamientos, funcionalidad de grupos científicos, especialización (áreas científico-técnicas, ciencias exactas, idiomas extranjeros) y estructuración de actividades. Lo que influyó en el desarrollo de redes internacionales, proyectos, transferencia tecnológica e innovación, así como también en el rigor respecto a las publicaciones científicas (Gungula; Artigas; Faustino, 2020; Gungula; Suárez; Artigas, 2020).

Los subprocesos de institucionalización, financiamientos, gestión de currículos, estructuración de claustros docentes, gestión de matrículas, armonización curricular, formaciones post-graduadas, estructuras físico-tecnológicas y productividad científica en el período 2005-2020 presentaron las insuficiencias anteriormente referidas; influyendo en la ejecución de los procesos investigativos. Lo cual afectó los niveles de planificación, organización, ejecución, control y evaluación de la investigación, donde además se manifestaron dificultades al gestionar las competencias investigativas desde sus concepciones y procedimientos teórico-metodológicos.

\section{Gestión de competencias investigativas en contextos universitarios angolanos}

La ejecución de los procesos investigativos en contextos universitarios precisan ser analizados además desde la gestión de competencias investigativas, donde se desarrollan acciones de planificación, organización, ejecución, control y evaluación dinámicamente, mediante el desarrollo de habilidades, destrezas, y hábitos, que determinan los desempeños al realizar actividades investigativas. Este análisis se sustentó en referentes teóricos, tales como: Benavides (2002), Fuentes (2004), Tobón (2005), Gayol, et al (2009), Ollarves; Salguero (2009), Londoño (2011), Cazares (2014) y Estrada (2014). 
Benavides (2002) definió las competencias investigativas, como requerimientos investigativos utilizados al indagar, monitorear, identificar necesidades, manejar informaciones estadísticas y realizar conclusiones sobre diversos aspectos analizados. Según Tobón (2005) constituyen construcciones del conocimiento científico para solucionar problemas, representando configuraciones psicológicas, proyecciones, ejecución, análisis, comunicación, introducción y generalización de los resultados investigativos.

No obstante Fuentes (2004), abordó otros aspectos teóricos relacionado con las competencias investigativas, al considerar los procesos investigativos como sistemas de acciones donde se ejecutan indagaciones, argumentación e innovación científica. Lo cual permite la configuración de disímiles subprocesos que se relacionan dialécticamente como un todo, dando lugar al surgimiento de categorías de órdenes superiores.

Por su parte, Cazares (2014) abordó la competencia investigativa como una interacción reflexiva y funcional establecida entre saberes, que genera actuaciones transferibles a distintos contextos, apoyadas por conocimiento situacional; ubicándose en una dimensión individual con capacidad de transmitirse hacia diferentes realidades. Mientras, Tobón (2005) apreció la competencia investigativa como desempeños (idoneidad, ética) que se desarrollan al resolver problemas, manifestando una realización personal.

No obstante otros referentes teóricos como Gayol, et al (2009) señalaron, que las competencias investigativa están integradas por capacidades cognoscitivas, donde se requiere manejar críticamente las bibliografías, delimitar el problema a investigar, abordar los trabajos individuales y grupales, formular hipótesis, precisar marco teórico, diseñar validaciones, así como también estructurar proyectos científicos.

Desde la anterior perspectiva cognitiva Ollarves; Salguero (2009), apreciaron además las competencias investigativas como destrezas, habilidades, conocimientos, comportamientos, actitudes y valores; que adecuadamente combinados frente a determinadas situaciones laborales, permiten desarrollar diversos desempeños estimuladores de productividad, calidad 0 innovación.

Igualmente, Londoño (2011) consideró que las competencias investigativas constituyen conjuntos de conocimientos, habilidades y actitudes desarrolladas desde la epistemología, metodología e implementación científica mediante técnicas. Lo cual estimula los niveles reflexivos, llegando a adquirir visibilidad y gestión del conocimiento.

Conjuntamente Fuentes (2004) reconoció las competencias investigativas como la síntesis del ser, saber, hacer y convivir desde ámbitos socio-culturales e intelectuales. Lo cual adquiere caracteres transversales donde se integran significados y sentidos para los sujetos implicados, siendo considerados como construcciones sociales e individuales,

Mientras Estrada (2014) abordó las competencias investigativas como una integración de la dimensión cognitiva (conocimientos, habilidades), a cualidades personales (actitudes, destrezas) y metacognitivas, que permiten desarrollar actividades indagativas, liderazgo, relaciones interpersonales, colaboración, actitud crítica, motivaciones, valores éticoprofesionales, así como también experiencias sociales.

Sustentado en los anteriores referentes teóricos el actual estudio aprecia el término competencia investigativa como acciones desarrolladoras de saberes (ser, saber, hacer, convivir). Las cuales se integran dinámicamente desde procesos indagativos, generando 
respuesta al encargo social mediante proyectos de investigación, socialización e implementación científica.

El actual estudio a partir de las anteriores definiciones analizó las dificultades, que se presentaron en las competencias investigativas para el desarrollo de los procesos investigativos en contextos universitarios angolanos; donde se manifestaron inadecuados procedimientos al interpretar e integrar informaciones teóricas y estadísticas; así como también en la argumentación científica.

Por otra parte, se identificaron irregularidades al planificar, organización, ejecutar, controlar y evaluar los procesos de investigación científica; lo cual ha sido abordado como etapas de gestión. El presente estudio analizó estas etapas teniendo en cuenta referentes tales como: Vidal; Araña (2012), Fuentes (2004) y Peluffo; Catalán (2016), quienes consideraron sus características estructurales como proceso.

Gestionar es sinónimo de administrar donde ocurre la planificación, implementación, control y evaluación de diferentes procesos en función del cumplimiento de determinados objetivos. Lo cual ha sido ampliado hacia subprocesos relacionados con la detección, selección, organización, filtrado, presentación y el uso de informaciones (Vidal; Araña, 2012).

No obstante, la gestión ha sido apreciada desde dimensiones cognitivas como estructuras, sistemas e interacciones conscientes diseñadas para dinamizar aprendizajes (conocimiento, habilidades). Por otro lado, al ejecutar investigaciones desde un carácter social se estimulan diferentes formaciones de competencias investigativas donde se crean, desarrollan y preservan talentos humanos conllevando a alcanzar los objetivos propuestos (Fuentes, 2004).

Por otra parte, las competencias investigativas al gestionarse deben transitar por estadios de desarrollo donde los conocimientos y tecnologías se sistematizan y se aplican dinámicamente mediante operaciones, procedimientos, formas 0 estructuras generando aprendizajes significativos (Peluffo; Catalán, 2016). Siendo un proceso caracterizado por aspectos teórico-metodológicos, que generan proactividad en el investigador desde roles sociales.

El actual estudio considera como gestión de competencias investigativas, a determinadas acciones que facilitan ejecutar dinámicamente las pesquisas 0 indagaciones científicas. Lo cual discurren al planificar, organizar, ejecutar, controlar y evaluar proyectos, metodologías, estrategias, programas, actividades, métodos, técnicas, operaciones 0 procedimientos; donde se desarrollan los diferentes saberes (ser, saber, hacer, convivir).

La gestión de competencias investigativas en contextos universitarios angolanos entre los años 2005 y 2020, manifestó dificultades al desarrollar acciones de planificación, organización, ejecución, control y evaluación dentro de los procesos investigativos. Lo cual fue motivado por irregularidades en las dinámicas de las indagaciones y socialización de los resultados investigativos (Gungula; Artigas; Faustino, 2020; Gungula; Suárez; Artigas, 2020).

Las indagaciones y socialización de los resultados investigativos presentaron irregularidades al abordar críticamente las bibliografías, delimitar problemas científicos, objetos y campos de actuación. Además persistieron las inconsistencias teórico-metodológicas en el desarrollo de las investigaciones; lo cual ha sido provocado por la falta de sistematización de los conocimientos, metodologías y tecnologías en los contextos universitarios.

Las referidas irregularidades teórico-metodológicas presentes en el desarrollo de las competencias investigativas desde contextos universitarios angolanos requieren de dinámicas, 
que permitan integrar dialécticamente los conocimientos, saberes, habilidades y actitudes. Lo cual proporcione aprendizajes significativos e implementaciones científicas, capaces de generar soluciones a las problemáticas contemporáneas emergidas de la sociedad actual.

\section{Conclusiones}

La revisión de los documentos seleccionados, posibilitó dar alcance al objetivo de analizar el basamento legal angolano en relación al desarrollo de la investigación y su implementación en contextos universitarios entre 2005 y 2020. Lo cual reveló una sistematicidad en los procesos legales, que posibilitaron trazar una política direccionada al desarrollo de la investigación.

La política ministerial hacia el desarrollo de la investigación desde contextos universitarios angolanos, estimuló el desarrollo de la ciencia e innovación; no obstante el desarrollo de la investigación científica presentó los desafíos de armonizar los procesos que permiten proyectar, implementar, socializar y generalizar las alternativas de solución a los problemas sociales y al desarrollo del país.

El desarrollo de la investigación en contextos universitarios angolanos precisa transitar sistemáticamente por procesos de planificación, organización, ejecución, control e valuación como una estructura de gestión, donde las competencias investigativas dinamicen las acciones y actividades investigativas, mediante la integración de saberes (ser, saber, hacer, convivir), que generen actividades cognitivo-intervencionistas desde proyectos, socialización e implementación científica.

De conformidad con lo anteriormente expresado, se concluye que las Instituciones de Educación Superior en contextos angolanos cuentan con un basamento legal, que estimula el desarrollo de la investigación en función del desarrollo del país, lo cual debe ser sustentado por el desarrollo de las competencias investigativas.

\section{Referencias Bibliográficas}

Angola (2011a). Política Nacional de Ciência, Tecnologia e Inovação (PNCTI). Decreto Presidencial N. ${ }^{\circ}$ 201/11, de 20 de Julho. Diário da República I Série- No.137. República de Angola.

Angola (2011b). Mecanismos de coordenação do Sistema Nacional de Ciência, Tecnologia e Inovação. Decreto Presidencial N. ${ }^{\circ} 224 / 11,11$ de Agosto de 2011, Diário da República I Série - N. ${ }^{0}$ 153. República de Angola.

Angola (2011c). Estrategia Nacional de Ciencia, Tecnología e Innovación. Decreto Presidencial N. ${ }^{\circ}$ 196/11, 11 de Julho de 2011, Diário da República I Série - N. ${ }^{\circ}$ 130. República de Angola.

Angola (2014). Reglamento del Consejo Superior de Ciencia, Tecnología e Innovación. Diário da República I Série N. $.^{\circ} 214,1$ de Dezembro de 2014. República de Angola.

Angola (2015a). Regulamento Geral dos Conselhos Científicos, Desenvolvimento Tecnológico e Inovação do Sistema Nacional de Ciência, Tecnologia e Inovação. Decreto Presidencial No.112/15, de 29 de Maio. Diário da República I Série- №78. República de Angola. 
Angola (2015b). Plano Anual de Ciência, Tecnologia e Inovação, 2 de Janeiro de 2015. Decreto Presidencial N. ${ }^{\circ} 1 / 15$ de 2 de Janeiro. Diário da República, I Série-N. ${ }^{\circ}$. República de Angola.

Angola (2015c). Regulamento Geral das Instituições Públicas de Investigação Científica, desenvolvimento tecnológico e inovação, 1 de Junho de 2015, Decreto Presidencial N. ${ }^{\circ}$ 125/15. Diário da República, I Série - N. ${ }^{\circ} 79$. República de Angola.

Angola (2016). Lei de Bases do Sistema de Educação e Ensino. Lei No17/16, 7 de Outubro. Diário da República, I Série- № 170. República de Angola.

Angola (2018). Estatuto de Carreira Docente Universitária. Decreto Presidencial No.191/18, de 8 de Agosto. Diário da República, I Série- No.118. República de Angola.

Angola (2020). Projecto de estatuto da Academia Angolana de Ciências, 21 de Janeiro de 2020. Diário da República, 21 de Janeiro de 2020. Diário da República III Série N. ${ }^{0} 14$. República de Angola.

Artigas, Wileidys y Gungula, Eurico (2020). Gestión de revistas a través de OJS: Experiencia de éxito Angolana. e-Ciencias de la Información, 10(1). https://doi.org/10.15517/eci.v10i1.39771

Assembleia Nacional (2001). Lei de Bases do Sistema de Educação. Lei No.13/2001, de 31 de Dezembro. Diário da República. República de Angola.

Benavides, Olga (2002). Competencias y competitividad. Diseño para Organizaciones Latinoamericanas. Ediciones McGRAW-HILL. Colombia.

Cazares, Solimar (2014). Planeación y Evaluación Basadas en Competencias. Extraído de https://pt.scribd.com/document/289060974/CAZARES-CUEVAS-2014-Planeacion-y-

Evaluacion-Basadas-en-Competencias

Conselho de Ministros (2009). Normas reguladoras do Subsistema de Ensino Superior. Decreto 90/09. Projecto Portal do Governo República de Angola.

Estrada, Odiel (2014). Sistematización teórica sobre la competencia investigativa. Revista Electrónica Educare. 18 (2). Costa Rica. (Pp. 177-194). Extraído de https://www.redalyc. org/articulo.oa?id=1941/194130549009

Fuentes, Homero (2004). El proceso de investigación científica desde un pensamiento dialéctico hermenéutico. Reto actual en la formación de doctores. Ed. Centro de estudios de Educación Superior Manuel F. Gran. Universidad de Oriente. Cuba.

Gayol, María; Montenegro, Silvana; Tarrés, María; D’Ottavio, Alberto (2009). Competencias Investigativas. Su desarrollo en carreras del Área de la Salud. Extraído de https://revistas.udea.edu.co/index.php/unip/article/view/950

Gungula, Eurico; Castillero, Josefina y Mendes, Maria (2017). Visibilidade dos Sistemas de Créditos Académicos nos Países Lusófonos. TELOS. Revista de Estudios Interdisciplinarios en Ciencias Sociales, 19 (2), 288-307. Extraído de: https://www.dialnet.unirioja.es/servlet/articulo?codigo $=6219234$

Gungula, Eurico; Artigas, Wileidys y Faustino, Arnaldo (2020). La difusión de la ciencia en Angola a través de revistas científicas: una alternativa de mejoramiento del proceso investigativo. Revista general de información y documentación, 30 (2). Extraído de: https://dx.doi.org/10.5209/rgid.72812 
Gungula, Eurico; Suárez, Wendolin y Artigas, Wileidys (2020). Investigar para Educar: Visões sem fronteiras. Luanda, Universidade Óscar Ribas/High Rate Consulting. Angola. DOI: https://doi.org/10.38202/Inv.educ

Londoño, Olga (2011). Desarrollo de la competencia investigativa desde los semilleros de investigación. Revista Científica General José María Córdova, 9 (9), Colombia. (Pp. 187-207). https://doi.org/10.21830/19006586.250

MESCTI (2019). Transferência de tecnologia e inovação em Angola. Progressos, fraquezas, desafios e oportunidade de melhoria. Luanda, Junho 2019. República de Angola.

Ministério da Educação e Cultura (2001). Estratégia integrada para a melhoria do sistema de educação, 2001-2015 Angola.

Extraído de https://planipolis.iiep.unesco.org/sites/planipolis/files/ressources/angola_estrategia_inte grada_melhoria.pdf

Ollarves, Yolibet; Salguero, Luis (2009). Una propuesta de competencias investigativas para los docentes universitarios. Laurus, Revista de Educación, 15 (30), Venezuela. (Pp. 118137). Extraído de: https://www.redalyc.org/761/76120651006

Ortiz Carrillo, José. (2021). Intercambio binacional en estudios de 4to nivel: caso Colombia y Venezuela. Revista Latinoamericana De Difusión Científica, 3(4), Colombia (Pp.2642). https://doi.org/10.38186/difcie.34.03

Peluffo, Martha y Catalán, Edith (2016). Introducción a la gestión del conocimiento y su aplicación al sector público. Edición № 21. Instituto Latinoamericano y del Caribe de Planificación Económica y Social - ILPES. Chile.

Secretaria de Estado do Ensino Superior (2005). Linhas Mestres, Subsistema de Ensino Superior. Extraído

https://planipolis.iiep.unesco.org/sites/planipolis/files/ressources/angola_linhas_mestras subsistema_ensino_superior.pdf

Tobón, Sergio. (2005). Formación basada en Competencias. Pensamiento complejo, diseño curricular y didáctica. Segunda edición. ECOE Ediciones. Colombia. Extraído de http://200.7.170.212/portal/images/documentos/formacion_basada_competencias.pdf

Vidal, María; Araña, Ana Bárbara (2012). Gestión de la información y el conocimiento. Revista Cubana de Educación Médica Superior, 26(3), Cuba. (Pp. 474-484). Extraído de http://scielo.sld.cu/pdf/ems/v26n3/ems13312. 